Math. Model. Nat. Phenom.

Vol. 5, No. 1, 2010, pp. 123-147

DOI: $10.1051 / \mathrm{mmnp} / 20105106$

\title{
Volume Filling Effect in Modelling Chemotaxis
}

\author{
D. Wrzosek * \\ Institute of Applied Mathematics and Mechanics, Warsaw University \\ Banacha 2, 02-097 Warszawa, Poland
}

\begin{abstract}
The oriented movement of biological cells or organisms in response to a chemical gradient is called chemotaxis. The most interesting situation related to self-organization phenomenon takes place when the cells detect and response to a chemical which is secreted by themselves. Since pioneering works of Patlak (1953) and Keller and Segel (1970) many particularized models have been proposed to describe the aggregation phase of this process. Most of efforts were concentrated, so far, on mathematical models in which the formation of aggregate is interpreted as finite time blow-up of cell density. In recently proposed models cells are no more treated as point masses and their finite volume is accounted for. Thus, arbitrary high cell densities are precluded in such description and a threshold value for cells density is a priori assumed. Different modeling approaches based on this assumption lead to a class of quasilinear parabolic systems with strong nonlinearities including degenerate or singular diffusion. We give a survey of analytical results on the existence and uniqueness of global-in-time solutions, their convergence to stationary states and on a possibility of reaching the density threshold by a solution. Unsolved problems are pointed as well.
\end{abstract}

Key words: chemotaxis equations, quasilinear parabolic equations, Lyapunov functional, degenerate diffusion, no-flux boundary condition, semi-group theory, compactness method, attractor, convergence to steady states, nonlinear elliptic problem.

AMS subject classification: Primary 35K55, 35K65 ; Secondary34B15, 34C25, 92C17

\footnotetext{
*E-mail: darekw@mimuw.edu.pl

* Supported by Polish MEiSW grant 1P03A01730
} 


\section{Introduction}

We give a survey of analytical results on a class of quasilinear systems of parabolic equations which are used to model the chemotactic motion of biological cells. Chemotaxis is understood here as a chemosensitive oriented movement of biological cells which may detect and respond to some signaling chemical (chemoattractant) secreted by their environment. The most interesting situation related to the self-organization phenomena, takes place when the cells themselves produce the signaling chemical which leads to the formation of cell aggregate [9]. It is further assumed that the total flux of cells consists of diffusive and chemotactic part

$$
J_{\text {tot }}=J_{\text {diff }}+J_{\text {chem }}
$$

The classical model describing the aggregation phase of chemosensitive motion of cells was introduced by Patlak [42] and Keller and Segel [31]. Most of works on Patlak/Keller-Segel model were focused on the case when cell diffusion is Brownian $J_{\text {diff }}=D \nabla u$ and chemotactic flux is proportional to the concentration gradient of chemical signal $J_{\text {chem }}=\chi u \nabla v$ where $u$ and $v$ denote the cell density and the concentration of chemoattractant respectively. The chemotactic sensitivity $\chi$ in the simplest case is a density-independent fixed constant. We thus obtain the so called minimal model of chemotaxis

$$
\begin{aligned}
\partial_{t} u & =\operatorname{div}\{D \nabla u-\chi u \nabla v\} \\
\partial_{t} v & =\Delta v+\gamma u-\beta v
\end{aligned}
$$

supplemented by initial condition and no flux boundary data. The second equation describes the rate of production $(\gamma)$ and degradation $(\beta)$ of diffusing chemoattractant.

Many results on the minimal model and related topics may be found in survey papers [28, 29] and [25]. The most interesting feature of the minimal model is the occurence of blow-up solutions in the space dimension $n=2$ provided the initial mass of cells is large enough. There is a vast literature on the blow-up phenomenon for the minimal model and for its elliptic-parabolic version (in (1.2) $\partial_{t} v$ replaced by 0 ) for which much more results are available. We mention here only results by Herrero and Velázquez [23, 24] and Nagai [36] where the existence of blowing-up solutions and the emergence of singularity in finite time is proved in the radially symmetric case. We also refer to [37] for the detailed study of singularity formation for a general domain in $\mathbb{R}^{2}$ as well as for indication of critical initial mass above which blow-up occurs. We note also that the emergence of singularity in finite time corresponds to the formation of the cell aggregate.

Recently new models which have nonlinearities in both chemotactic and diffusive part have been introduced. Among them our attention in this survey is focused on those in which the variable describing cell population is a priori bounded.

Accordingly to one approach due to the finite size of cells and volume limitations the density of cells is assumed to be bounded by the threshold value normalized to 1 and corresponding to the tight packing state. This concept was first developed by Hillen and Painter [26, 41] (volume filling model of chemotaxis (VF)) and can be also included in the frame of hydrodynamical model (HM) of chemotaxis introduced in [32]. 
The second approach is based on the concept of two phase modeling. Byrne and Owen proposed a two-phase model (TP) describing cells moving through a fluid containing a diffusible chemoattractant. Now variable $u$ denotes a volume fraction of cells in a two component mixture that consists of cells and fluid (e.g. water) with volume fraction $w=1-u$. The derivation of the model is based on mass and momentum balance and some additional constitutive laws that describe interaction between cells, fluid and chemoattractant. Another model in which $u$ is interpreted as a volume fraction has recently been derived in Lushnikov et al. [35] as a macroscopic limit of cellular Potts model (MLCPM) which accounts for stochastic system of finite size cells with exclusion volume principle.

On the one hand, derivation and interpretation of the models are based on different approaches, but on the other one they turn out to share some common mathematical features. Both diffusive and chemotactic part contain here density dependent nonlinearities which lead in some cases to degenerate or singular diffusion. Moreover, by different reasons the variable describing the population of cells is a priori bounded which preclude the blow-up of solution as a feature indicating the appearance of cells aggregate. Instead of it, a solution which attains the threshold value 1 indicates regions where cells are tightly packed. A corresponding pattern formation mechanism was investigated for (VF) in [26, 41]. Existence of flat-hump-shaped stationary solutions such that cells density attain the threshold value on some set with non-empty interior was recently proved in [33] and in [52].

More precisely, all models considered here can be viewed in the following abstract form

$$
\begin{aligned}
\partial_{t} u & =\operatorname{div}\{D(u) E(u, v) \nabla u-u \chi(u, v) \nabla v\}, \\
\partial_{t} v & =d \Delta v+g(u, v),
\end{aligned}
$$

in $(0, \infty) \times \Omega$ subjected to the no-flux boundary condition

$$
\langle D(u) E(u, v) \nabla u-u \chi(u, v) \nabla v \mid \nu\rangle=\langle\nabla v \mid \nu\rangle=0 \quad \text { on } \quad(0, \infty) \times \partial \Omega,
$$

and the initial condition

$$
(u, v)(0)=\left(u_{0}, v_{0}\right) \text { in } \Omega,
$$

where $\Omega$ is a bounded open subset of $\mathbb{R}^{n}$ with smooth boundary $\partial \Omega$ and $\nu$ is an outward unit normal vector on the boundary. Here, $u$ is interpreted in (VF) as the cell density or as a volume fraction in (TP). Next, $v$ is a chemoattractant concentration, $D(u) E(u, v)$ denotes the density-dependent diffusion coefficient and $\chi(u, v)$ is usually named chemotactic sensitivity function. The function $g$ describes the rates of production and degradation of the chemoattractant $v$. Without loss of generality diffusion constant in (1.4) is normalized to 1 . In particular, we note that the minimal model of chemotaxis corresponds to the case where $D, E$ and $\chi$ are constants. In the next sections we present in detail four types of aforementioned models and then give a survey of their qualitative properties.

The way in which the cell density threshold (normalized to 1) is built in varies from model to model. In the models (VF), (TP) and (HM) it is assumed that the diffusion and chemotaxis drop away up to the threshold i.e $D(1)=\chi(1, v)=0$. On the other hand in (MLCPM) just the diffusivity is assumed to blow up at the threshold. 
In this paper we do not consider in detail those works in which the volume filling effect is implicit, so that there is no any a priori limit on the density of cells involved (see $[12,13,14$, $15,16,48,54]$. The papers consider various assumptions on nonlinear diffusion and chemotactic sensitivity function which lead to the existence of global in time solutions, thus preventing blowup.

\subsection{Volume filling model (VF).}

The volume filling model (VF) was formally derived in [41] from a discrete space, continuous time, reinforced random walk on an equi-distant lattice. Let $u=u(x, t)$ denotes the cell density at a position $x$ and time $t$ then $q(u(x, t))$ is introduced in [41] to determine the probability that a cell in a position $x$ and time $t$ finds space at its neighbouring location. Then $q=q(s)$ is a given non-negative and non-increasing function such that $q^{\prime}(s)<0$ for $s \in(0,1]$. According to another interpretation proposed in [49] $q(u)$ is named squeeze probability. Because of the volume filling effect in the tight packing state normalized here as $u \equiv 1$ we have $q(1)=0$. Consequently, it turns out that the chemotactic component of the total flux attains zero and the chemotactic movement stops when $u=1$. We then have

$$
D(r)=D_{0}\left(q(r)-r q^{\prime}(r)\right), \quad E \equiv 1 \quad \text { and } \quad \chi(r, s)=\chi_{0} q(r), \quad r \in[0,1] \times[0,+\infty)
$$

where $D_{0}$ and $\chi_{0}$ are positive constants. The latter is called the chemosensitivity constant. Further properties of the system depend on the assumptions imposed on $q$. Thus, we obtain the following possibilities for cell diffusion:

$$
\begin{array}{cl}
q(u)=1-u & \text {-linear diffusion } \\
q^{\prime}(1)<0 & \text {-quasilinear diffusion } \\
q^{\prime}(1)=0 & \text {-degenerate diffusion } \\
q^{\prime}(1)=-\infty & \text {-fast(singular) diffusion }
\end{array}
$$

If cells are treated as solid blocks then $\mathrm{q}(\mathrm{u})$ is proportional to the numbers of occupants and hence linearly dependent on the cell density as in(1.8). Then $D=D_{0}$ and $\chi(r, s)=\chi_{0}(1-r)$. We may consider two locations of graph of $q(u)$

- The case when $q(u)>1-u$ may be interpreted as the ability of cells to change (or adapt) their configurations to squeeze into an open space. The sqeeze probability is then higher than that for the case of solid blocks. As an example it may be taken $q(u)=1-u^{\gamma}$ with $\gamma>1$. The "limiting case" for this situation is when (1.11) holds. If we assume for instance $q(s)=(1-s)^{r}$ with $r \in(0,1)$ then fast diffusion appears in (VF) model and we refer to [49] for basic properties of this model and numerical solutions. The corresponding system reads

$$
\begin{aligned}
\partial_{t} u & =\nabla \cdot \overbrace{\left\{D_{1} \frac{1-(1-r) u}{(1-u)^{1-r}}\right.}^{D(u)} \nabla u-u \overbrace{\chi_{0}(1-u)^{r}}^{h(u)} \nabla v\}, \\
\partial_{t} v & =\Delta v+\gamma u-\beta v .
\end{aligned}
$$


Existence of global in time weak solutions to this system was proved in [53] (see Section 2.1, and Section 2.2).

- The case when $q(u)<1-u$ corresponds to the situation when crowding or packing effects slow down both diffusive and chemotactic movement of cells. This results in nonlinear decreasing diffusion as well as chemotactic sensitivity. The "limiting case " is when (1.10) holds, thus the diffusion becomes degenerate at $u=1$. For instance for $q(u)=(1-u)^{2}$ we obtain $D(u)=(1-u)(1+u)$.

The family of volume-filling chemotaxis models with the density threshold increasing to the infinity $q(u)=\varepsilon^{-1}-u$ was used in [47] to approximate the minimal model (1.1)-(1.2) as $\varepsilon \rightarrow 0$ and to study formation of singularities at the blow-up time and to construct solutions after the blowup.

We mention also paper [11] in which convergence in the drift-diffusion limit from a kinetic model for chemotaxis to the (VF) model with (1.8) is proved. On the other hand thanks to the $L^{\infty}$ bound resulting from the volume-filling assumption, the non-diffusive limit of (1.1)-(1.2) as $D \rightarrow 0$ is performed in [19]. In the case of one space dimension it is proved there that the solution to (VF) model converges to the entropy solution to the corresponding hiperbolic-parabolic system $(D=0$ in (1.1)-(1.2)). Similar result was proved in [43] where the entropy solution to a hyperbolic-elliptic system is obtained in the limit of solution to the parabolic-elliptic (VF) model with (1.8).

\subsection{Hydrodynamical model (HM).}

We next present a hydrodynamical approach to chemotaxis equations proposed in [32]. Population of cells is treated now as a non-viscous fluid with density $u$ and velocity $\mathbf{v}$ which satisfies the Euler equations

$$
\begin{aligned}
\partial_{t} u+\nabla \cdot(u \mathbf{v}) & =0 \\
u\left(\partial_{t} \mathbf{v}+\mathbf{v} \cdot \nabla \mathbf{v}\right) & =-\nabla p+F(u, v, \mathbf{v})
\end{aligned}
$$

with the density-dependent pressure $p=p(u)$ and the force

$$
F=\chi_{0} \nabla v-\beta(u) \mathbf{v}
$$

composed from the chemotactic part oriented toward the gradient of the chemoattractant $v$ and a resistive force which is due to all factors moderating cell movement in particular kinematic friction. We note that originally $\beta$ is taken as a constant friction coefficient in [32]. Then following [32] we assume that inertial force is negligible for the description of cells movement and we obtain

$$
\chi_{0} \nabla v-\nabla p(u)-\beta(u) \mathbf{v}=0 .
$$

Hence, using (1.14) we arrive at

$$
\partial_{t} u=\nabla \cdot\left(\frac{u p^{\prime}(u)}{\beta(u)} \nabla u-\frac{\chi_{0} u}{\beta(u)} \nabla v\right)
$$


If we suppose that the probability of finding space is, due to crowding, inversely proportional to the frictional drag forces i.e $q(u)=\beta(u)^{-1}$ and set

$$
p(u)=\ln (u \beta(u)) \quad u \in(0,1)
$$

it is easy to check that for such a choice of $q$ and $p$ in (1.16) we obtain the (VF) model with

$$
D(u)=\beta(u)^{-1}-u \overbrace{\left(\beta^{\prime}(u) \beta(u)^{-2}\right)}^{q^{\prime}(u)} .
$$

It shows that derivations of chemotaxis equations based on entirely different approaches lead to mathematical models which are linked at least on a formal level.

\subsection{Two-phase model (TP), two-point degenerate diffusion}

Recently Byrne and Owen [10] proposed a two-phase model describing cells moving through a fluid containing a diffusible chemoattractant. Now variable $u$ denotes a volume fraction of cells in a two component mixture that consists of cells and fluid (e.g. water) with volume fraction $w=1-u$. The derivation of the model is based on the mass and momentum balance and some additional constitutive laws that describe interaction between cells, fluid and chemoattractant. Then changes in cells volume fraction are governed by (1.3) with the following substitution

$$
D(r)=\frac{r(1-r)^{2}}{k(r)}, \quad E=\Lambda(r, s)+r \partial_{r} \Lambda(r, s) \quad \text { and } \quad \chi(r, s)=\frac{r(1-r)^{2}}{k(r)} \partial_{s} \Lambda(r, s)
$$

for $(r, s) \in[0,1] \times[0,+\infty)$ where $k=k(r)$ is a drag function and $\Lambda(r, s)$ is a potential function which determines cells behavior. If one assumes diffusion to be a dominant factor causing the movement of chemoattractant molecules and neglect advection then fluctuation of chemoattractant in fluid may be described by an equation of the form (1.4). We call system (1.3) with (1.17) and (1.4) a simplified version (TPs) of full (TP) model which originally takes also into account the advection term in chemoattractant equation. The latter term leads, however, in general to inverse diffusion problem and even if one then restricts to a degenerate parabolic case it turns out to be non-triangular with gradient dependent reaction terms. This property leads to serious problems in proving well-posedness of global -in-time solutions.

\subsection{Macroscopic limit to the cellular Potts model (MLCPM).}

A more recent model introduced in [35], is obtained as a macroscopic limit of microscopic cellular dynamics of finite size cells with exclusion volume principle (meaning that two cells can not occupy the same volume) accounted for. The diffusivity in the latter model belongs also to the class of singular diffusion and $h$ and $E$ in (1.3)-(1.6) are constants. For the one dimensional case $(n=1)$ a system of type (1.3)-(1.6) is obtained with the following first equation

$$
\partial_{t} u=\nabla \cdot\left(\frac{D_{2}\left(1+u^{2}\right)}{1-u)^{2}} \nabla u-\chi u \nabla v\right) .
$$


For the case $(n=2)$ the first equation reads

$$
\partial_{t} u=\nabla \cdot\left(\frac{D_{3}(1+u)}{1-u+u \log u} \nabla u-\chi u \nabla v\right) .
$$

In both cases the second equation is the same as in (1.2) and no-flux boundary conditions are accounted for. Parameters $D_{2}$ and $D_{3}$ are positive constants. It is easy to check that in this model diffusion operator is singular which is refered to as superdiffusion according to Aronson's classification [6]

$$
D(u)=\frac{D_{3}(1+u)}{1-u+u \log u}>\frac{D_{3}}{(1-u)^{2}}, \quad u \in(0,1) .
$$

\section{Existence and uniqueness of solutions}

We shall first present how the existence of global in time solutions in the case of non-degenerate diffusion may be derived from the theory of quasilinear parabolic systems developed by Amann $[4,5]$. Next we shall present the concept of weak solutions which applies to both degenerate and singular diffusion problems.

\subsection{Classical solutions}

In general the existence of classical solutions is not expected for degenerate or singular parabolic problems. Still, in such cases classical solutions usually exist for related regularized problems and may be used for aproximation of weak solutions. The existence and uniqueness of classical solutions was proved in [26, 41, 50, 33]for regular parabolic case of (VF) (1.8)-(1.9). The existence of classical solutions may be deduced from the abstract theory of quasilinear parabolic systems developed by Amann. This theory besides the local existence of solutions provides criteria for the existence of global-in-time solutions.

We adhere to usual notation of function spaces (cf. [1] ); $W^{m, p}\left(\Omega ; \mathbb{R}^{n}\right)$ for $m \geq 1,1<p<$ $+\infty$, denotes Sobolev space of $\mathbb{R}^{n}$-valued functions equipped with the usual norm $\|\cdot\|_{m, p}$. The norm in the Lebesgue space $L^{p}(\Omega), 1 \leq p \leq+\infty$ is denoted by $|\cdot|_{p}$.

The following theorem concerns the situation when

$$
E \equiv 1
$$

in (1.3)-(1.5) and $g \in \mathcal{C}^{1}\left(\mathbb{R}^{2}\right)$ satisfies

$$
g(r, 0) \geq 0 \text { and } \partial_{s} g(r, s) \leq \kappa \text { for }(r, s) \in[0,1] \times[0, \infty),
$$

for some $\kappa>0$ along with

$$
\begin{array}{rlll}
\chi=h(r)>0 & \text { for } & r \in(0,1), & \text { and } h(1)=0 \\
D(r)>0 & \text { for } & r \in[0,1) . &
\end{array}
$$


The theory of Amann demands the following regularity assumptions

$$
D \in \mathcal{C}^{2}(\mathbb{R}), \quad \text { and } h \in \mathcal{C}^{2}(\mathbb{R}) .
$$

As for the initial data, we assume that

$$
\left(u_{0}, v_{0}\right) \in L^{\infty}\left(\Omega ; \mathbb{R}^{2}\right) \text { with } 0 \leq u_{0} \leq 1 \text { and } 0 \leq v_{0} \text { a.e. in } \Omega .
$$

The proof of the following theorem can be easily extended to the case when functions $D$ and $h$ have more general form. As an example of such extension we refer to [52] where the well-posedness of regularized version of problem (1.3)-(1.5) is considered.

Theorem 1. [33] Let $p>N$ and consider $\left(u_{0}, v_{0}\right) \in W^{1, p}\left(\Omega ; \mathbb{R}^{2}\right)$ such that (2.6) holds true. Let $g$, $h$, and $D$ be functions satisfying (2.3) (2.2) and (2.4),(2.5), and assume further that there is $\eta>0$ such that

$$
D(r) \geq \eta \quad \text { for } \quad r \in[0,1] .
$$

Then there is a unique classical solution

$$
(u, v) \in \mathcal{C}\left([0,+\infty) \times \bar{\Omega} ; \mathbb{R}^{2}\right) \cap \mathcal{C}^{1,2}\left((0,+\infty) \times \bar{\Omega} ; \mathbb{R}^{2}\right)
$$

to (1.3)-(1.6) such that $(u(t, x), v(t, x)) \in[0,1] \times[0,+\infty)$ for each $(t, x) \in[0,+\infty) \times \bar{\Omega}$. In addition,

$$
\int_{\Omega} u(t, x) d x=\int_{\Omega} u_{0}(x) d x \text { for each } t \geq 0 .
$$

Proof. We put $D_{0}:=(-\delta,+\infty) \times(-\delta, 1+\delta)$ and denote by $\mathcal{M}_{2}(\mathbb{R})$ the space of $2 \times 2$ real valued matrices. Next we define $a \in \mathcal{C}^{2}\left(D_{0} ; \mathcal{M}_{2}(\mathbb{R})\right)$ by

$$
a(y)=\left(a^{m, n}\right)_{1 \leq m, n \leq 2}:=\left(\begin{array}{cc}
1 & 0 \\
-y_{2} h\left(y_{2}\right) & D\left(y_{2}\right)
\end{array}\right) \quad \text { for } \quad y=\left(y_{1}, y_{2}\right) \in D_{0} .
$$

We set $a_{j, k}(y):=a(y) \delta_{j, k}$ for $1 \leq j, k \leq N$ where $\delta_{j, k}$ is the Kronecker symbol and $y \in D_{0}$. We next introduce the operators

$$
\begin{aligned}
& \mathcal{A}(y) z:=-\sum_{j, k=1}^{N} \partial_{j}\left(a_{j, k}(y) \partial_{k} z\right), \\
& \mathcal{B}(y) z:=\sum_{j, k=1}^{N} \nu_{j} \cdot a_{j, k}(y) \partial_{k} z
\end{aligned}
$$

and the function

$$
f(y)=\left(f^{m}\right)_{1 \leq m \leq 2}:=\left(\begin{array}{c}
g\left(y_{2}, y_{1}\right) \\
0
\end{array}\right)
$$


with $z=\left(z_{1}, z_{2}\right)$. Then (1.3)-(1.6) reads in the following abstract form

$$
\begin{aligned}
\partial_{t} z+\mathcal{A}(z) z & =f(z) \\
\mathcal{B}(z) z & =0 \\
z(0) & =\left(v_{0}, u_{0}\right)
\end{aligned}
$$

with $z=(v, u)$. The eigenvalues of the matrix $a(y)$ are positive for each $y \in D_{0}$, and the boundaryvalue operator $(\mathcal{A}, \mathcal{B})$ is of separated divergence form in the sense of [5, Example 4.3 (e)]. Consequently, the boundary-value operator $(\mathcal{A}, \mathcal{B})$ is normally elliptic by [5, Section 4]. We are then in a position to apply [5, Theorems $14.4 \& 14.6]$ to conclude that (1.3)-(1.6) has a unique maximal classical solution

$$
z:=(v, u) \in \mathcal{C}\left(\left[0, T_{\star}\right) \times \bar{\Omega} ; \mathbb{R}^{2}\right) \cap \mathcal{C}^{1,2}\left(\left(0, T_{\star}\right) \times \bar{\Omega} ; \mathbb{R}^{2}\right),
$$

where $T_{\star} \in(0,+\infty]$. In addition, for $y_{1}, y_{2} \geq 0$, we have $a^{1,2}\left(0, y_{2}\right)=0, a^{2,1}\left(y_{1}, 0\right)=0$ and $f^{1}\left(0, y_{2}\right)=g\left(y_{2}, 0\right) \geq 0$ by (2.2). These properties along with (1.6) and [5, Theorem 15.1] ensure that $v(t, x) \geq 0$ and $u(t, x) \geq 0$ for $(t, x) \in\left[0, T_{\star}\right) \times \bar{\Omega}$. We next observe that $\tilde{z}:=(v, 1-u)$ is a solution to a similar initial-boundary value problem with

$$
\tilde{a}(y)=\left(\tilde{a}^{m, n}\right)_{1 \leq m, n \leq 2}:=\left(\begin{array}{cc}
1 & 0 \\
-h\left(1-y_{2}\right) y_{2} & D\left(1-y_{2}\right)
\end{array}\right) \quad \text { for } \quad y=\left(y_{1}, y_{2}\right) \in D_{0} .
$$

Since $\tilde{a}^{2,1}\left(y_{1}, 0\right)=-h(1)=0$, a further application of [5, Theorem 15.1] and (2.6) yield that $1-u(t, x) \geq 0$ for $(t, x) \in\left[0, T_{\star}\right) \times \bar{\Omega}$. So far, we have thus proved that

$$
0 \leq u(t, x) \leq 1 \quad \text { and } \quad 0 \leq v(t, x)
$$

for $(t, x) \in\left[0, T_{\star}\right) \times \bar{\Omega}$. Also, integrating (1.3) over $(0, t) \times \Omega$ implies that (2.8) holds true for $t \in\left[0, T_{\star}\right)$. We finally infer from (2.2) that

$$
\partial_{t} v-\Delta v \leq g(u, 0)+\kappa v \leq C(1+v)
$$

with $C:=\|g(., 0)\|_{L^{\infty}(0,1)}+\kappa$, from which we conclude by comparison that

$$
v(t, x) \leq\left(C+\left\|v_{0}\right\|_{L^{\infty}}\right) e^{C t}, \quad(t, x) \in\left[0, T_{\star}\right) \times \bar{\Omega} .
$$

It is worth to mention at this point that the matrix $a$ in(2.9) is lower-triangular and one could use [5, Theorem 15.5] to conclude that $T_{\star}=+\infty$.

The assumption (2.5) may be relaxed to the form $D \in \mathcal{C}^{2}(0,1)$ and $h \in \mathcal{C}^{2}(0,1)$ and then similar arguments lead (see [49] and [53]) to the existence of local-in-time classical solutions to (VF) model with fast diffusion (1.11). So far it is not known whether global in time solutions exist in this case. We note that it would be sufficient to prove that $u(t, \cdot)$ is separated from 1 for any time interval $[0, T] \subset\left[0, T_{\star}\right)$. On the other hand the existence of global-in-time weak solutions which may attain the threshold value 1 for this case was proved in [53]). 
At last we mention that the existence of global-in-time classical solutions to (MLCPM) model was proved independently in [2] and in [53]. Moreover in the latter paper a general system is considered

$$
\begin{aligned}
\partial_{t} u & =\nabla \cdot(D(u) \nabla u-u h(u) \nabla v), \\
\partial_{t} v & =\Delta v+\gamma u-\beta v
\end{aligned}
$$

wit the following set of assumptions:

$$
D, h:(-\delta, 1) \mapsto \mathbb{R}, D, h \in \mathcal{C}^{2}(-\delta, 1) \text { for some } \delta>0,
$$

there is a constant $C_{D}>0$ such that for $r \in(-\delta, 1)$

$$
D(r) \geq C_{D}(1-r)^{-\alpha} \text { for } \alpha>0,
$$

there is a constants $C_{h}>0$ such that for $r \in(-\delta, 1)$

$$
h(r)>0 \text { and } h(r) \leq C_{h}(1-r)^{\beta} \text { for } \beta \geq 0 .
$$

The case $\alpha=1-s, \beta=s$ for $s \in(0,1)$ corresponds to (VF) model with fast diffusion (1.11) and the case of $\alpha=2, \beta=0$ refers to Model (MLCPM) with superdiffusion $(\alpha>1)$ ). Notice that the larger is the value of $\beta$ the weaker the impact of chemotactic force on the movement of cells when their density is close to the threshold value.

It is proven in [53] that global-in-time classical solution to (1.1)-(1.2) exists provided

$$
\beta \geq 1-\frac{\alpha}{2}
$$

and that the global solution is uniformely separated from the threshold 1 whenever $\beta>2$ with arbitrary $\alpha>0$. It is worth mentioning that both papers [2] and [53] exploit some ideas from [17] where the existence of global solutions to system (2.11)-(2.12) with the assumptions $h(u) \equiv 1$ and $D(u)=(1-u)^{-\alpha}$ for $\alpha \geq 2$ and $n \geq 2$ is proved.

\subsection{Weak solutions}

The concept of a weak solution to the chemotaxis equations (1.3)-(1.6) with degenerate diffusion was first used in [33] where it was assumed $D(1)=h(1)=0$ with (2.3)-(2.4) and $E \equiv 1$. Then it was extended in [52] for the case of a system including model (TP) and independently for chemotaxis model with two sidedly degenerate diffusion [7]. Recently the concept was used for the case of fast diffusion of cells [53] including (VF) model (1.12)-(1.13). In order to introduce the concept of weak solution we first define

$$
\mathcal{D}(r):=\int_{0}^{r} D(s) d s, \quad r \in[0,1] .
$$


Definition 2. Let $\left(u_{0}, v_{0}\right) \in L^{\infty}\left(\Omega ; \mathbb{R}^{2}\right)$ be such that (2.6) holds true. A weak solution to (1.3)-(1.6) is a couple $(u, v)$ of functions such that, for each $T>0$,

$$
\begin{gathered}
0 \leq u(t, x) \leq 1, \quad 0 \leq v(t, x) \quad \text { a.e.in }(0, T) \times \Omega \\
u \in L^{\infty}((0, T) \times \Omega) \cap \mathcal{C}_{w}\left([0, T] ; L^{2}(\Omega)\right), \quad \mathcal{D}(u) \in L^{2}\left(0, T ; H^{1}(\Omega)\right), \quad u(0)=u_{0} \\
v \in L^{\infty}((0, T) \times \Omega) \cap H^{1}\left(0, T ; L^{2}(\Omega)\right) \cap L^{2}\left(0, T ; H^{2}(\Omega)\right), \quad v(0)=v_{0}
\end{gathered}
$$

and $(u, v)$ satisfy

$$
\begin{aligned}
\int_{0}^{T}\left\langle\partial_{t} u, \psi\right\rangle d t & +\int_{0}^{T} \int_{\Omega}(E(u, v) \nabla \mathcal{D}(u)-u \chi(u, v) \nabla v) \nabla \psi(t, x) d x d t=0, \\
\partial_{t} v & =\Delta v+g(u, v) \text { a.e.in }(0, T) \times \Omega, \\
\partial_{\nu} v & =0 \text { a.e. on }(0, T) \times \partial \Omega,
\end{aligned}
$$

for each $t \in[0, T]$ and $\psi \in L^{2}\left(0, T ; H^{1}(\Omega)\right)$. Here, $\langle.,$.$\rangle denotes the duality pairing between$ $H^{1}(\Omega)$ and $H^{1}(\Omega)^{\prime}$.

We denoted by $\mathcal{C}_{w}\left([0, T] ; L^{2}(\Omega)\right)$ the set of functions from $[0, T]$ in $L^{2}(\Omega)$ which are continuous for the weak topology of $L^{2}(\Omega)$. The functions $D \in \mathcal{C}^{2}(\mathbb{R}), \chi \in \mathcal{C}^{2}(\mathbb{R})$ satisfy

$$
\begin{array}{cl}
D(0) \geq 0, D(1) \geq 0 \quad \text { and } \quad D(r)>0 & \text { for } r \in(0,1), \\
\chi(1, s)=0, \quad \chi(r, s)>0 & \text { for }(r, s) \in(0,1) \times[0,+\infty) .
\end{array}
$$

Notice that we are mainly interested in the degenerate case when

$$
D(0)=0 \text { and/or } D(1)=0 .
$$

We also assume that $e_{0}>0$ such that

$$
E(r, s) \geq e_{0} \text { for all }(r, s) \in(0,1) \times[0,+\infty),
$$

and that the reaction term $g \in \mathcal{C}^{2}\left(\mathbb{R}^{2}\right)$ satisfies the (one-sided) growth condition (2.2). We note that assumptions (2.24), (2.25), (2.2) and (2.27) comprise most of examples studied in [41] and [10]. In particular, we may have in (1.17) $k(r)=$ const, $k(r)=(1-r), k(r)=r(1-r)$ and $\Lambda(r, s)=\alpha+\gamma \exp \left\{-\frac{\theta s}{1-r}\right\}$ (cf. [10]). As it was mentioned in the Introduction classical solutions are expected to exist for the case when the elliptic part of the operator is non-degenerate and weak solutions are involved when the diffusion operator is degenerate or singular.

In the degenerate case of (VF) model both diffusive and chemotactic parts of cells flux tend to 0 along with cells density tending to the threshold value. Existence and uniqueness of solutions to (VF) model in the degenerate case (1.10) follows from [33].

Theorem 3. [52] Consider $\left(u_{0}, v_{0}\right) \in L^{\infty}\left(\Omega ; \mathbb{R}^{2}\right)$ such that (2.6) holds true. Under the assumptions (2.24)-(2.27) and (2.2) on D, $\chi$ and $g$, there exists a weak solution (u,v) to (TP) model (1.3)-(1.6) such that

$$
\int_{\Omega} u(t, x) d x=\int_{\Omega} u_{0}(x) d x \text { for each } t \geq 0
$$


The method of proof relies on the suitable regularization of the original problem and on the compactness method. The regularized problem is strongly parabolic and its solvability may be proved in the similar way as for Theorem 1 in the previous section. At last we mention the article [7] where the existence of weak solutions for chemotaxis equations with two-sidedly (twopoint) degenerate diffusion of cells was shown independently of [52] by means of slightly different method. In [3] the existence of weak solutions to predator prey system with prey taxis is proved. In this model predator plays formally the role of cells in the chemotaxis model and prey plays that of chemoattractant, the reaction terms reflect prey-predator interactions and the density of prey is assumed to be bounded due to the volume filling effects.

\subsection{Uniqueness of weak solutions}

Uniqueness of weak solutions was first proved in [33] for the chemotaxis equations with degenerate diffusion satisfying (2.1), (2.3), (2.4) and $D(1)=0$ under further assumptions on $D, h$ and $g$ : there are $C_{0}>0, C_{1}>0$ and functions $g_{1} \in \mathcal{C}^{2}(\mathbb{R})$ and $g_{2} \in \mathcal{C}^{2}(\mathbb{R})$ such that $g_{1}(0) \geq 0, g_{2}(0) \geq 0$,

$$
\begin{aligned}
& (r h(r)-s h(s))^{2} \leq C_{0}(r-s)(\mathcal{D}(r)-\mathcal{D}(s)), \\
& g(r, s)=g_{1}(s)+r g_{2}(s) \quad \text { and } \quad \max \left\{g_{1}^{\prime}(s), g_{2}^{\prime}(s)\right\} \leq C_{1},
\end{aligned}
$$

for $(r, s) \in[0,1] \times[0,+\infty)$, where $\mathcal{D}(r)$ is defined in (2.17). The proof of uniqueness relies on the duality method. Then this approach was extended for two-point degenerate diffusion [52] and for the case of fast diffusion [53] of cells. A sufficient condition on $h$ and $D$ which guarantees that (2.29) holds true is the following

Proposition 4. If $h^{\prime} D^{-1 / 2} \in L^{\infty}(0,1)$, then (2.29) holds true.

In particular, the functions $D(r)=(1-r)^{m}$ and $h(r)=(1-r)^{n}$ satisfy (2.3) and (2.29) if $m \geq(n+2) / 2$ and $n \geq 2$.

However, the uniqueness problem for (TP) model requires, in the general case, a new method since that from [33] can only be adopted here if $E(r, s)$ does not depend on $s$ variable and $\chi$ is factorized $\chi(r, s)=\chi_{1}(r) \chi_{2}(s)$.

\section{Stationary states}

In this and subsequent sections, we assume the particular linear form

$$
g(r, s):=\gamma r-\beta s
$$

for some $\beta>0$ and $\gamma>0$ and that (2.1), (2.4), (2.3) are satisfied. Extensive study of stationary solutions to non-degenerate chemotaxis equations was performed in [45]. We concentrate here on the case of chemotaxis models with degenerate diffusion of cells (see [33]) i.e. (VF) model with (1.10) and (TP) model. The study of stationary solutions for the case of two-point degenerate diffusion of cells is done in [52] for some particular cases. 
Definition 5. [33] (i) A stationary solution to (1.3)-(1.5) with the assumptions (2.1), (2.4), (2.3) is a couple $(u, v)$ of functions in $L^{\infty}\left(\Omega ; \mathbb{R}^{2}\right)$ such that $\mathcal{D}(u) \in H^{1}(\Omega), v \in H^{2}(\Omega)$,

$$
\begin{aligned}
& (u(x), v(x)) \in[0,1] \times[0,+\infty) \text { a.e. in } \Omega, \\
& \nabla \mathcal{D}(u)-u h(u) \nabla v=0 \text { a.e. in } \Omega, \\
& -\Delta v+\beta v=\gamma u \text { a.e. in } \Omega, \\
& \partial_{\nu} v=0 \text { a.e. on } \partial \Omega .
\end{aligned}
$$

(ii) If $M \in[0,|\Omega|]$, a stationary solution to (1.3)-(1.5) with mass $(M, \gamma M / \beta)$ is a stationary solution $(u, v)$ to (1.3)-(1.5) satisfying in addition

$$
\int_{\Omega} u(x) d x=\frac{\beta}{\gamma} \int_{\Omega} v(x) d x=M .
$$

Obviously, $(u, v)=(0,0)$ and $(u, v)=(1, \gamma / \beta)$ are the only stationary solutions to (1.3)(1.5) with mass $(0,0)$ and $(|\Omega|, \gamma|\Omega| / \beta)$, respectively. Also, if $m \in(0,1)$ then $(m, \gamma m / \beta)$ is a stationary solution (1.3)-(1.5) with mass $(m|\Omega|, \gamma m|\Omega| / \beta)$. The stability of constant stationary solutions to the (VF) model with linear diffusion (1.8) in one space dimension was studied in [44]. Moreover it is shown there by means of phase method ( cf. Section 3.2 in the sequel) that there are non-constant stationary states in this case. We next describe some properties of stationary solutions with mass $(M, \gamma M / \beta)$ for $M \in(0,|\Omega|)$. For further use, we introduce the auxiliary functions

$$
j(r):=\int_{1 / 2}^{r} \frac{D(s)}{s h(s)} d s \text { and } J(r):=\int_{1 / 2}^{r} j(s) d s, \quad r \in(0,1),
$$

which enjoy the following property.

Lemma 6. The function $j$ is an increasing function from $(0,1)$ onto $\left(-\infty, j_{1}\right)$ where

$$
j_{1}:=\int_{1 / 2}^{1} \frac{D(s)}{\operatorname{sh}(s)} d s \in(0,+\infty] .
$$

Proof. The strict monotonicity of $j$ follows at once and for $s \in(0,1 / 2]$,

$$
\frac{D(s)}{\operatorname{sh}(s)} \geq \frac{\min _{[0,1 / 2]} D}{\|h\|_{L^{\infty}(0,1)}} \frac{1}{s} \geq \frac{C}{s}
$$

for some $C>0$, so that $D / h$ does not belong to $L^{1}(0,1 / 2)$.

The following result provides general information on the shape of the stationary solutions.

Proposition 7. [33] Let $M \in(0,|\Omega|)$ and consider a stationary solution $(u, v)$ to (1.3)-(1.5) with mass $(M, \gamma M / \beta)$. Then $\Omega_{u}:=\{x \in \Omega, u(x) \in[0,1)\}$ is a non-empty open subset of $\Omega, j(u)-v$ is constant on each connected component of $\Omega_{u}$ and $u \in \mathcal{C}(\bar{\Omega}) \cap \mathcal{C}^{1}\left(\Omega_{u}\right)$. In addition, $u(x)>0$ for $x \in \bar{\Omega}$ and

$$
v(x) \in\left[0, \frac{\gamma}{\beta}\right], \quad x \in \bar{\Omega} .
$$


Proof. Since $u \in L^{\infty}(\Omega)$, we infer from (3.4) and (3.5) that $v \in W^{2, p}(\Omega)$ for every $p \in(1, \infty)$. Choosing $p$ large enough, we realize that $\nabla v \in \mathcal{C}(\bar{\Omega})$, which, together with (3.2) and (3.3), implies that $\mathcal{D}(u) \in W^{1, \infty}(\Omega)$. Consequently, $\mathcal{D}(u) \in \mathcal{C}(\bar{\Omega})$ and, since $\mathcal{D}$ is increasing, we have also $u \in \mathcal{C}(\bar{\Omega})$. A further use of (3.3) then yields that $\mathcal{D}(u) \in \mathcal{C}^{1}(\bar{\Omega})$. Therefore, recalling that $u \geq 0$, we deduce that $\Omega_{u}$ is an open subset of $\Omega$ (which is non-empty by (3.2) since $M<|\Omega|$ ) and $u \in \mathcal{C}^{1}\left(\Omega_{u}\right)$ with $\nabla \mathcal{D}(u)=D(u) \nabla u$ on $\Omega_{u}$.

Now, let $\Lambda$ be a connected component of $\{x \in \Omega, u(x) \in(0,1)\}$. For $x \in \Lambda$, (3.3) also reads $\nabla(j(u)-v)(x)=0$. Therefore, there is $\lambda \in \mathbb{R}$ such that $j(u(x))=v(x)+\lambda$ for each $x \in \Lambda$. Thanks to the monotonicity of $j$, we have in fact $u(x)=j^{-1}(v(x)+\lambda) \geq j^{-1}(\lambda)>0$ for $x \in \Lambda$. The continuity of $u$ then entails that $u(x) \geq j^{-1}(\lambda)>0$ for $x \in \bar{\Lambda}$. Consequently, $u(x)>0$ for $x \in \bar{\Omega}$ and $\Omega_{u}=\{x \in \Omega, u(x) \in(0,1)\}$. It then follows from (3.3) that $\nabla(j(u)-v)=0$ on $\Omega_{u}$.

Finally, the bounds (3.9) are straightforward consequence of (3.4) and (3.5) by the comparison principle.

Accounting for (3.8) we distinguish two cases:

- non-degenerate case when $j_{1}=+\infty$,

- degenerate case when $j_{1}<+\infty$.

\subsection{The non-degenerate case}

We next derive some upper and lower bounds for stationary solutions which generalize [51, Proposition 1.4].

Proposition 8. [33] Let $M \in(0,|\Omega|)$ and consider a stationary solution $(u, v)$ to (1.3)-(1.5) with mass $(M, \gamma M / \beta)$. Assume further that $j_{1}=+\infty$. Then

$$
j^{-1}\left(j\left(\frac{M}{|\Omega|}\right)-\frac{\gamma}{\beta}\right) \leq u(x) \leq j^{-1}\left(j\left(\frac{M}{|\Omega|}\right)+\frac{\gamma}{\beta}\right), \quad x \in \bar{\Omega} .
$$

In particular, $u(x) \in(0,1)$ for $x \in \bar{\Omega}$.

Proof. Let $\Lambda$ be a connected component of $\Omega_{u}$. By Proposition 7, there is $\lambda \in \mathbb{R}$ such that

$$
j(u(x))=v(x)+\lambda \text { for } x \in \Lambda .
$$

Since $j_{1}=+\infty$, we infer from (3.9) that $u(x) \leq j^{-1}((\gamma / \beta)+\lambda)<1$, from which we conclude that $\Lambda=\Omega_{u}=\Omega$ by the continuity of $u$. Therefore,

$$
M=\int_{\Omega} u(x) d x=\int_{\Omega} j^{-1}(v(x)+\lambda) d x \in\left[|\Omega| j^{-1}(\lambda),|\Omega| j^{-1}\left(\frac{\gamma}{\beta}+\lambda\right)\right],
$$

whence

$$
\lambda \in\left[j\left(\frac{M}{|\Omega|}\right)-\frac{\gamma}{\beta}, j\left(\frac{M}{|\Omega|}\right)\right] .
$$


The claimed bounds then follow from (3.9), (3.10) and the above estimates for $\lambda$.

When $D$ and $h$ are related as in (VF) model, we have

$$
j(r)=\frac{1}{\chi_{0}}(\ln (2 r)-\ln (q(r))+\ln (q(1 / 2))
$$

and $D / h \notin L^{1}(1 / 2,1)$. In particular when $q(u)=1-u$ then

$$
j(r)=\frac{1}{\chi_{0}} \ln \frac{r}{1-r}
$$

\subsection{The degenerate case}

In contrast to the previous case where we have shown that the first component $u$ of any steady state to (1.3)-(1.5) cannot reach the threshold value 1 (unless $u \equiv 1$ ) it was proved in [33] and then extended for two- point degenerate model that in the one dimensional case there are stationary solutions $(u, v)$ to (1.3)-(1.5) such that $u=1$ on some subset of $\Omega$ with non-empty interior, so that the graph of $u$ is flat-hump-shaped. To this end, we recall that, by Proposition 7, $j(u)-v$ is constant on each connected component of $\Omega_{u}=\{x \in \Omega, u(x) \in[0,1)\}$ and look for a stationary solution $(u, v)$ such that the constant does not depend on the connected component of $\Omega_{u}$. Introducing $f:=j^{-1}$ and $f_{\lambda}():.=f(.+\lambda)$ for $\lambda \in \mathbb{R}$, we thus look for a stationary solution $(u, v)$ to (1.3)(1.5) such that there is $\lambda \in \mathbb{R}$ for which $u(x)=f_{\lambda}(v(x))$ for $x \in \Omega_{u}$. Since $u(x)=1$ for $x \in \Omega \backslash \Omega_{u}$, we actually have

$$
u(x)=F_{\lambda}(v(x)) \text { for } x \in \bar{\Omega},
$$

where

$$
F_{\lambda}(y)=\left\{\begin{array}{cl}
f_{\lambda}(y) & \text { for } y \leq v_{\lambda}:=j_{1}-\lambda \\
1 & \text { for } y>v_{\lambda}
\end{array}\right.
$$

Next, (3.4) and (3.11) yield that

$$
-\Delta v=\gamma F_{\lambda}(v)-\beta v \text { in } \Omega
$$

with no-flux boundary condition on $\partial \Omega$. Observe that the continuity of $v$ established in Proposition 7 and (3.13) imply that

$$
\begin{aligned}
-\Delta v & =\gamma-\beta v \quad \text { in } \Omega_{0}:=\Omega \backslash \bar{\Omega}_{u}, \\
v & =v_{\lambda} \text { on } \partial \Omega_{0} .
\end{aligned}
$$

We finally recall that (3.9) warrants that

$$
v_{\lambda} \leq \gamma / \beta
$$

A sufficient condition for the existence of a flat-hump-shaped stationary solution to (1.3)-(1.5) is explained below. To this end, we say that the triple $(\lambda, \gamma, \beta) \in \mathbb{R} \times(0,+\infty)^{2}$ satisfies the condition $(\mathbf{C})$ if 


$$
(\mathbf{C})\left\{\begin{aligned}
\text { There are } \varrho_{-1}, \varrho_{0}, \varrho_{1} & =\gamma / \beta \text { such that } \varrho_{-1}<\varrho_{0}<v_{\lambda}<\varrho_{1} \text { and } \\
F_{\lambda}\left(\varrho_{i}\right) & =\frac{\beta}{\gamma} \varrho_{i}, \quad i \in\{-1,0,1\} \\
F_{\lambda}(y) & >\frac{\beta}{\gamma} y, \quad y \in\left(\varrho_{0}, \varrho_{1}\right) \\
F_{\lambda}(y) & <\frac{\beta}{\gamma} y, \quad y \in\left(\varrho_{-1}, \varrho_{0}\right)
\end{aligned}\right.
$$

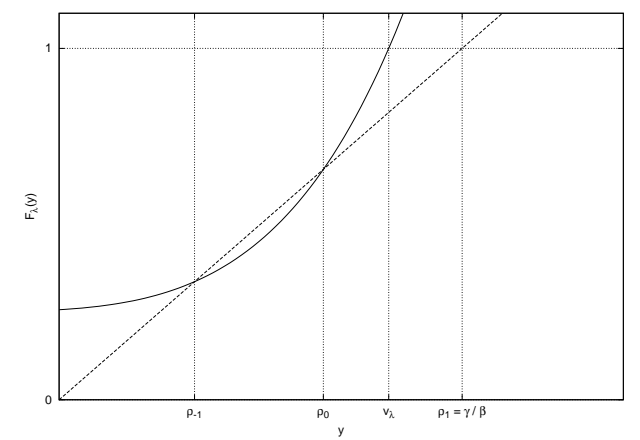

Figure 1: Possible location of the graph of $F_{\lambda}$ for which condition (C) is satisfied.

Theorem 9. [33] Suppose that there is a triple $(\lambda, \gamma, \beta)$ which satisfies the condition $(\mathbf{C})$ together with

$$
\frac{1}{v_{\lambda}-\varrho_{-1}} \int_{\varrho_{-1}}^{v_{\lambda}} f_{\lambda}(y) d y<\frac{\beta}{\gamma} \frac{v_{\lambda}+\varrho_{-1}}{2} .
$$

Then, for l large enough, there exists a flat-hump-shaped stationary solution $(u, v)$ to (1.3)-(1.5) in $\Omega=(0, l)$. More precisely, there exists $x_{1} \in(0, l / 2)$ such that $u(x) \in[0,1)$ for $x \in\left[0, x_{1}\right) \cup$ $\left(l-x_{1}, l\right], u(x)=1$ for $x \in\left[x_{1}, l-x_{1}\right]$ and $v(x) \geq v_{\lambda}$ for $x \in\left[x_{1}, l-x_{1}\right]$.

If additionally

$$
f^{\prime} \in L^{2}\left(j_{1}-\delta, j_{1}\right) \quad \text { for some } \delta>0,
$$

then $u \in H^{1}(0, l)$.

The proof of this theorem is based on phase plane method applied to the one-dimensional boundary-value problem

$$
\begin{aligned}
v^{\prime \prime} & =\Phi(v) \quad \text { in }(0, l), \quad v^{\prime}(0)=v^{\prime}(l)=0, \\
\Phi(v) & =-\gamma F_{\lambda}(v)+\beta v .
\end{aligned}
$$

which is equivalent to the hamiltonian system

$$
v^{\prime}=w, \quad w^{\prime}=\Phi(v),
$$




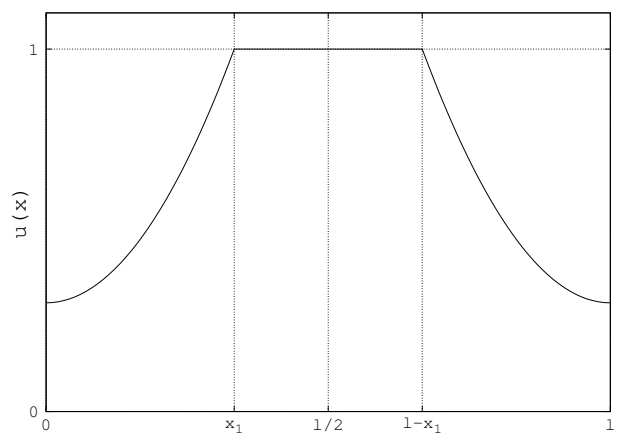

Figure 2: Flat-hamp-shaped stationary solution.

with "energy" $\mathcal{E}$ and "potential" $\mathcal{U}$ given by

$$
\mathcal{E}(v, w)=\frac{1}{2} w^{2}+\mathcal{U}(v), \quad \mathcal{U}(v)=-\int_{\varrho_{0}}^{v} \Phi(y) d y .
$$

It turns out that the condition (3.17) is not restrictive.

Proposition 10. Suppose that the triple $(\lambda, \gamma, \beta)$ satisfies the condition $(\mathbf{C})$ and (3.17) is not satisfied. Then there is $\lambda_{2}$ such that $\left(\lambda_{2}, \gamma, \beta\right)$ satisfies $(\mathbf{C})$ and (3.17) holds.

Proof. We shall denote the intersection points of the graph of $f_{\lambda}$ with the graph of the function $y \mapsto \frac{\beta}{\gamma} y$ by $\varrho_{-1}(\lambda)$ and $\varrho_{0}(\lambda)$. If $(\lambda, \gamma, \beta)$ satisfies the condition $(\mathbf{C})$ then for some $\lambda_{1}<\lambda$ we have $v_{\lambda_{1}}=\varrho_{1}=\frac{\gamma}{\beta}$. In this case

$$
f_{\lambda_{1}}(y)<\frac{\beta}{\gamma} y \text { for } y \in\left(\varrho_{-1}(\lambda), \varrho_{1}\right)
$$

Hence, for mean values

$$
\frac{1}{v_{\lambda_{1}}-\varrho_{-1}\left(\lambda_{1}\right)} \int_{\varrho_{-1}\left(\lambda_{1}\right)}^{v_{\lambda_{1}}} f_{\lambda_{1}}(y) d y<\frac{1}{v_{\lambda_{1}}-\varrho_{-1}\left(\lambda_{1}\right)} \int_{\varrho_{-1}\left(\lambda_{1}\right)}^{v_{\lambda_{1}}} \frac{\beta}{\gamma} y d y=\frac{\beta}{\gamma} \frac{v_{\lambda_{1}}+\varrho_{-1}\left(\lambda_{1}\right)}{2} .
$$

Now using the continuity argument we infer that there is $\lambda_{2} \in\left(\lambda_{1}, \lambda\right)$ such that $\left(\lambda_{2}, \gamma, \beta\right)$ still satisfies the condition (C) and (3.17) holds with $\lambda=\lambda_{2}$.

Remark 11. (i) It follows from the proof of the above theorem that, for $\lambda, \gamma, \beta$ satisfying the assumptions of Theorem 9, there exists a unique (singular) solution such that $u$ is spike-shaped (it suffices to choose the unique $v_{0} \in\left(\varrho_{-1}, \varrho_{0}\right)$ such that $\mathcal{U}\left(v_{0}\right)=\mathcal{U}\left(v_{\lambda}\right)$. In that case $\Omega_{u}=$ $(0, l) \backslash\{l / 2\}$.

(ii) It is possible to merge a finite number of flat-hump-shaped stationary solutions to obtain a multi-hump solution on a suitably enlarged interval.

(iii) It is possible to modify the proof of Theorem 9 to construct a stationary hump-shaped solution which is not symmetric with respect to $l / 2$. To this end one should choose two different values of 
$\lambda$ on each connected component of the set $\Omega_{u}$. Proceeding as in the proof of Theorem 9 one can construct separately a part of the solution defined on a subinterval containing 0 and another part on a subinterval containing $l$. Then one can merge both in such a way that, at a merging point $x_{0}$, we have

$$
\lim _{x \rightarrow x_{0}^{-}} v_{x}(x)=0=\lim _{x \rightarrow x_{0}^{+}} v_{x}(x)
$$

and there is a possible jump discontinuity of $v_{x x}$ at $x_{0}$.

We now specify conditions under which Theorem 9 can be applied. We first show that the condition (C) is valid under a simple condition on $j^{\prime}$ or $f^{\prime}$ near $j_{1}$.

Proposition 12. If

$$
\lim _{y \rightarrow j_{1}^{-}} f^{\prime}(y)>\frac{\beta}{\gamma}
$$

then there is $\varepsilon>0$ such that the triple $(\lambda, \beta, \gamma)$ satisfies the condition $(\boldsymbol{C})$ for any $\lambda \in\left(j_{1}-\right.$ $\left.\gamma / \beta, j_{1}+\varepsilon-\gamma / \beta\right)$.

Proposition 13. Consider $(\beta, \gamma) \in(0,+\infty) \times(0,+\infty)$. Assume that $D / h$ has a limit $j_{2}$ in $[0, \infty]$ as $r \rightarrow 1$ such that

$$
j_{2}>\frac{\gamma}{\beta}
$$

and that $(D / h)^{\prime \prime}(r)<0$ for every $r \in[0,1)$ such that $(D / h)^{\prime}(r)=0$. Introducing

$$
r_{0}:=\sup \left\{r \in(0,1), j^{\prime}(r)=\frac{\gamma}{\beta}\right\}
$$

the triple $(\lambda, \gamma, \beta)$ satisfies the condition $(\boldsymbol{C})$ if

$$
j_{1}-\frac{\gamma}{\beta}<\lambda<j\left(r_{0}\right)-\frac{\gamma}{\beta} r_{0} .
$$

As a final remark, let us point out that, in the case $h(r)=r D(r)$, a flat-hump-shaped stationary solution $(u, v)$ to (1.3)-(1.5) coincides with a stationary solution to the so-called minimal chemotaxis model in which $D \equiv 1$ and $h(r)=r$ (cf. [28]) on the set $\{x: u(x)<1\}$.

At last we mention two-point degenerate case when $D(r)=r h(r)$, It is proved in [52] that $j$ is then an affine function with $j(0)<+\infty, j(1)<+\infty$ and then non-homogeneous stationary solutions exist in arbitrary space dimension provided the domain of definition has some specific properties related to eigenvalue problem for the Laplace operator with the homogeneous Neumann boundary condition [52, Theorem 6]. In 1D case several types of stationary solutions including flat-hump shaped ones are distinguished. In [52, Theorem 8] the classification of stationary states for this case is provided. 


\section{Long time behaviour of solutions}

\subsection{Existence of a global attractor.}

Let us define the operator

$$
A_{r} \varphi=-\Delta \varphi+\varphi
$$

for

$$
\varphi \in\left\{w \in W^{2, r}: \frac{\partial w}{\partial \nu}=0\right\} .
$$

Then $A_{r}$ is a sectorial operator for which fractional powers $A^{\alpha}, \alpha \in(0,1)$, are well defined. The $\alpha$-norm of a vector $\varphi$ in the space $X_{r}^{\alpha}=\operatorname{Dom}\left(A_{r}^{\alpha}\right)$ (cf. [22] )will be denoted by $\|\varphi\|_{X_{r}^{\alpha}}$ for $\alpha \in(0,1)$. Next we define the set

$$
V_{1}=\left\{w=\left(w_{1}, w_{2}\right) \in W^{1, p}\left(\Omega, \mathbb{R}^{2}\right) ; 0 \leq w_{1}(x) \leq 1, \quad 0 \leq w_{2}(x) \text { for } x \in \Omega\right\}
$$

In this and the next subsection we consider (VF) model with

$$
q(u)=1-u .
$$

At first we admit in the first equation a growth term $f \in C^{2}(\mathbb{R}: \mathbb{R})$ which corresponds to the proliferation with food limitation in the population of cell. Thus we assume that there exists $K<1$ and $f(u) \leq 0$ for $x \in(K, 1)$ and $f(u)>0$ for $u \in(0, K)$. Then we consider

$$
\begin{aligned}
& \frac{\partial u}{\partial t}=\operatorname{div}\left\{\nabla u-\chi_{0} u(1-u) \nabla v\right\}+u f(u), \\
& \frac{\partial v}{\partial t}=\Delta v+\gamma u-\beta v,
\end{aligned}
$$

in $\Omega \times(0,+\infty)$ subject to initial and no-flux boundary conditions.

It is proved in [50] that $\left(S_{t}, V_{1}\right)$ is a dynamical system defined by the nonlinear semigroup

$$
S_{t} w_{0}=w\left(t, w_{0}\right), \quad t \geq 0 \quad w_{0} \in V_{1} .
$$

where $w\left(\cdot, w_{0}\right)$ is the solution to (4.2)-(4.3). We refer a reader to the well known monograph [46] for the definition of a global attractor.

The following theorem is proved in [50].

Theorem 14. Suppose that $w\left(t, w_{0}\right)$ is a solution to (4.2)-(4.3) starting from $w_{0} \in V_{1}$. Then i) there exists $t_{0}>0$ such that for $t>t_{0}$

$$
w(t) \in X_{r}^{\alpha_{0}} \times X_{r}^{\alpha_{0}}
$$

where $r>\max \left(p, \frac{n p}{p-n}\right)$ and $\alpha_{0} \in\left(\frac{1}{2}+\frac{n}{2 r}, 1-\frac{n}{2 p}+\frac{n}{2 r}\right)$. Moreover there exists a constant $C$ independent of initial data such that

$$
\limsup _{t \rightarrow \infty}\|w(t)\|_{X_{r}^{\alpha_{0}} \times X_{r}^{\alpha_{0}}} \leq C .
$$

ii) dynamical system $\left(S_{t}, V_{1}\right)$ possesses a global attractor. 
It is worth to note that for a range of parameters the attractor is non-trivial since it contains unstable stationary state [50]. The diffusion in both equations and the degradation in the second one are key factors which provide enough dissipativeness to the system such that the global attractor exists. We refer to $[40,38,39]$ for results on the existence and properties of global attractor for the minimal chemotaxis equations enriched with a source term which in this case playes a crucial role preventing the blow-up of solutions and providing the dissipativeness to the system.

\subsection{Convergence to the stationary state}

The study of the long time behavior of solutions to the chemotaxis equations is based on Lyapunov functional which has to be constructed for every particular model. This approach was used in [8] and [21] for the minimal model of chemotaxis (1.1)-(1.2). We mention also [27] where the Lyapunov functional for some class of chemotaxis equations is derived. Let us recall the definition of the function $J$ in (3.7). In [51] it is proved that

$$
\mathcal{V}(u, v)=\int_{\Omega} J(u) d x-\int_{\Omega} u v d x+\frac{1}{2 \gamma} \int_{\Omega}|\nabla v|^{2} d x+\frac{\beta}{2 \gamma} \int_{\Omega} v^{2} d x
$$

defined for $(u, v) \in V_{1}$ is a Lyapunov functional for the dynamical system $\left(S_{t}, V_{1}\right)$. It is worth noticing that under assumption (4.1) we obtain the particular form

$$
\int_{\Omega} J(u) d x=\frac{1}{\chi_{0}} \int_{\Omega}\{u \ln u+(1-u) \ln (1-u)-\ln 1 / 2\} d x
$$

and in this case Lyapunov functional has an explicit form. It is worth noticing that in the case of Patlak/Keller-Segel model

$$
\int_{\Omega} J(u) d x=\frac{1}{\chi_{0}} \int_{\Omega}\{u \ln u-\ln 1 / 2\} d x
$$

Theorem 15. For any $\bar{w} \in V_{1}$

$$
\mathcal{V}(\bar{w})>-\frac{\gamma}{\beta}|\Omega|
$$

and moreover for any $t>s \geq 0$

$$
\mathcal{V}(w(t))+\frac{1}{\chi_{0}|\Omega|} \int_{s}^{t}\left(\int_{\Omega}|D(u) \nabla u-h(u) \nabla v| d x\right)^{2} d \sigma+\frac{1}{\gamma} \int_{s}^{t} \int_{\Omega} v_{t}^{2} d x d \sigma \leq \mathcal{V}(w(s))
$$

where $w=(u, v)$ is a solution such that $u_{0}(x) \neq 0$ for all $x \in \Omega$.

Notice that the compactness of trajectories may be deduced from Theorem 14 and embedding theorems. Let us denote by $\omega\left(w_{0}\right)$ the $\omega$-limit set of a trajectory starting from $w_{0} \in V_{1}$. The following characterization of the $\omega$-limit set is proved in [50]. 
Theorem 16. If (4.1) is satisfied then $\omega\left(w_{0}\right)$ is a nonempty set and each couple $(U, V) \in \omega\left(w_{0}\right)$ is a stationary solution. Moreover $(U, V) \in C^{2, \sigma}(\bar{\Omega}) \times C^{2, \sigma}(\bar{\Omega})$ for some $\sigma \in(0,1)$. If $(U, V) \neq$ $(0,0)$ then for some $\lambda \geq 0$ it satisfies the following nonlocal problem

$$
\begin{aligned}
U & =\frac{1}{1+\lambda \exp \left\{-\chi_{0} V\right\}}, \\
-\Delta V+\beta V & =\frac{\gamma}{1+\lambda \exp \left\{-\chi_{0} V\right\}} \\
\int_{\Omega} U(x) d x & =\int_{\Omega} u_{0}(x) d x
\end{aligned}
$$

with no-flux boundary condition for (4.8).

Remark 17. We note that (4.7) may be rewritten in the following way

$$
U=\frac{1}{1+\lambda \exp \left\{-\chi_{0} V\right\}}=\frac{\lambda^{-1} \exp \left\{\chi_{0} V\right\}}{\lambda^{-1} \exp \left\{\chi_{0} V\right\}+1} .
$$

Then it is easy to check that the first component of the stationary solution to the minimal model model of chemotaxis $(q(u)=1)$ satisfies a similar equation as that above. The only difference is the denominator in the last formula which is equal to 1 in the case of minimal chemotaxis model.

Recently, Łojasiewicz-Simon inequality was used in [30] to prove that the solution to (VF) model with (4.1) starting from $\left(u_{0}, v_{0}\right)$ converges to one of the steady states in the set $\omega\left(u_{0}, v_{0}\right)$. Similar result was first proved for the minimal chemotaxis model (1.1)-(1.2) by Feireisl et al. [20].

At last but not least we mention the paper [18] in which the long time behavior of solutions to (VF) model with linear diffusion (1.8) is studied in all space i.e. $\Omega=\mathbb{R}^{n}$. Under basic assumption that diffusion constant is big enough with respect to chemosensitivity constant $\left(D>\frac{1}{4}\right.$ when $\chi_{0}=1$ ) the decay estimates for the solutions are proved in the space $L^{2}\left(\mathbb{R}^{n}\right)$ for any $n \geq 1$. The decay estimates in $L^{\infty}\left(\mathbb{R}^{n}\right)$ norm are proved only for $n=1$ and this result enables to show that in this case the asymptotic profile of solution is Gaussian.

\section{References}

[1] R. A. Adams. Sobolev spaces. Academic Press, New York, 1975.

[2] M. Alber,R. Gejji, B. Kaźmierczak. Existence of global solutions of a macroscopic model of cellular motion in a chemotactic field. Applied Mathematics Letters., 22 (2009), No. 11, 1645-1648.

[3] B. Ainsebaa, M. Bendahmaneb, A. Noussairc. A reaction-diffusion system modeling predator-prey with prey-taxis. Nonlinear Anal. R. World Appl., 9 (2008), No. 5, 2086 - 2105. 
[4] H. Amann. Dynamic theory of quasilinear parabolic systems III. Global existence. Math. Z., 202 (1989), No. 2, 219-250.

[5] H. Amann. Nonhomogeneous linear and quasilinear elliptic and parabolic boundary value problems.9-126, in: (H. Triebel, H.J. Schmeisser., eds.), Function Spaces, Differential Operators and Nonlinear Analysis. Teubner-Texte Math., 133, Teubner, Stuttgart, 1993.

[6] D. G. Aronson. The porous medium equation., in: (A.Fasano, M.Primicerio.,eds.) Some Problems in Nonlinear Diffusion. Lecture Notes in Mathematics., 1224, Springer, Berlin, 1986.

[7] M. Bendahmane, K. H. Karlsen, J. M. Urbano. On a two-sidedly degenerate chemotaxis model with volume-filling effect. Math. Models Methods Appl. Sci., 17 (2007), No. 2, $783-$ 804.

[8] P. Biler. Local and global solvability of some parabolic systems modelling chemotaxis. Adv. Math. Sci. Appl. Nachr., 195 (1998), No. 8, 76-114.

[9] M. P. Brenner, L. S. Levitov and E. O. Budrene. Physical mechanism for chemotactic pattern formation by bacteria. Biophys. J., 74 (1998), No. 4, 1677-1693.

[10] H. M. Byrne, M. R. Owen. A new interpretation of the Keller-Segel model based on multiphase modelling. J. Math. Biol., 49 (2004), No. 6, 604-626.

[11] F. A. C. C. Chalub, J. F. Rodrigues. A class of kinetic models for chemotaxis with threshold to prevent overcrowding. Portugaliae Math., 26 (2006), No. 2, 227-250.

[12] V. Calvez, J. A. Carillo. Volume effects in the KellerSegel model: energy estimates preventing blow-up. J. Math. Pures Appl., 86 (2006), No. 2, 155-175.

[13] T. Cieślak. The solutions of the quasilinear Keller-Segel system with the volume filling effect do not blow up whenever the Lyapunov functional is bounded from below. 127-132, in: Selfsimilar solutions of nonlinear PDE, Banach Center Publ., 74, Warsaw, 2006.

[14] T. Cieślak. Quasilinear nonuniformly parabolic system modelling chemotaxis. J. Math. Anal. Appl., 326 (2007), No. 2, 1410-1426.

[15] T. Cieślak, C. Morales-Rodrigo. Quasilinear non-uniformly parabolic-elliptic system modelling chemotaxis with volume filling effect. Existence and uniqueness of global-in-time solutions. Topol. Methods Nonlinear Anal., 29 (2007), No. 2, 361-381.

[16] T. Cieślak, M. Winkler. Finite-time blow-up in a quasilinear system of chemotaxis. Nonlinearity., 21 (2008), No. 5, 1057-1076.

[17] Y. S. Choi, Z. A. Wang. Prevention of blow up by fast diffusion in chemotaxis. J. Math. Anal. Appl., 362 (2010), No. 2, 553-564. 
[18] M. DiFrancesco, J. Rosado. Fully parabolic Keller-Segel model for chemotaxis with prevention of overcrowding. Nonlinearity., 21 (2008), No. 11, 2715-2730.

[19] Y. Dolak, C. Schmeiser. The Keller-Segel model with logistic sensitivity function and small diffusivity. SIAM J. Appl. Math., 66 (2005), No. 1, 286-308.

[20] E. Feireisl, Ph. Laurençot, H. Petzeltova. On convergence to equilibria for the Keller-Segel chemotaxis model. J.Diff.Equations., 236 (2007), No. 2, 551-569.

[21] H. Gajewski, K. Zacharias. Global behavior of a reaction-diffusion system modelling chemotaxis. Math. Nachr., 195 (1998), No. 1, 77-114.

[22] D. Henry. Geometric Theory of Semilinear Parabolic Equations. Lecture Notes in Mathematics, Springer-Verlag, Berlin, 1981.

[23] M. A. Herrero, J. J. L Velázquez. A blow-up mechanism for a chemotaxis model. Ann. Scuola Norm. Sup. Pisa., 24 (1997), No. 4, 633-683.

[24] M. A. Herrero, J. J. L Velázquez. Chemotactic collapse for the Keller-Segel model. J. Math. Biol., 35 (1996), No. 2, 583-623.

[25] T. Hillen, K. J. Painter. A user's guide to PDE models for chemotaxis. J. Math. Biol., 58 (2009), No. 1-2, 183-217.

[26] T. Hillen and K. Painter. Global existence for a parabolic chemotaxis model with prevention of overcrowding. Adv. Appl. Math., 26 (2001), No. 4, 280-301.

[27] D. Horstmann. Lyapunov functions and $L^{p}$-estimates for a class of reaction-diffusion systems. Colloq. Math., 87 (2001), No. 1, 113-127.

[28] D. Horstmann. From 1970 until present: the Keller-Segel model in chemotaxis and its consequences. I. Jahresber. Deutsch. Math.-Verein., 105 (2003), No. 3, 103-165.

[29] D. Horstmann. From 1970 until present: the Keller-Segel model in chemotaxis and its consequences. I. Jahresber. Deutsch. Math.-Verein., 106 (2004), No. 2, 51-69.

[30] J. Jiang, Y. Zhang. On Convergence to equilibria for a Chemotaxis Model with Volume filling effect. Asymptotic Analysis., 65 (2009), No. 1-2, 79-102.

[31] E. Keller and L. Segel. Initiation of slime mold aggregation viewed as an instability. J. Theor. Biology. 26 (1970), No. 3, 399-415.

[32] R. Kowalczyk, A. Gamba and L. Preciosi. On the stability of homogeneous solutions to some aggregation models. Discrete Contin. Dynam. Systems-Series B. 4 (2004), No. 1, 204-220.

[33] Ph. Laurençot, D. Wrzosek. A chemotaxis model with threshold density and degenerate diffusion. 273-290 in: Progress in Nonlinear Differential Equations and Their Applications., 64, Birkhäuser, Basel, 2005. 
[34] J.-L. Lions. Quelques méthodes de résolution des problèmes aux limites non linéaires. Dunod, Paris, 1969.

[35] P. M. Lushnikov, N. Chen and M. Alber. Macroscopic dynamics of biological cells interacting via chemotaxis and direct contact. Phys. Rev. E., 78 (2008), No. 6, 061904.

[36] T. Nagai. Blow-up of radially symetric solutions to a chemotaxis system. Adv. Math. Sci. Appl., 5 (1995), No. 2, 581-601.

[37] T. Nagai, T. Senba, T. Suzuki. Chemotaxis collapse in a parabolic system of mathematical biology. Hiroshima Math. J., 30 (2000), No. 3, 463-497.

[38] K. Osaki, A. Yagi. Finite dimensional attractors for one dimensional Keller-Segel equations. Funkcial. Ekvac., 44 (2001), No. 3, 441-469.

[39] K. Osaki, A. Yagi. Global existence for a chemotaxis-growth system in $\mathbb{R}^{2}$. Adv. Math. Sci. Appl., 12 (2002), No. 2, 587-606.

[40] K. Osaki, T. Tsujikawa, A. Yagi, M. Mimura. Exponential attractor for a chemotaxis-growth system of equations. Nonlinear Anal., 51 (2002), No. 1, 119-144.

[41] K. Painter and T. Hillen, Volume-filling and quorum-sensing in models for chemosensitive movement. Canadian Appl. Math. Q., 10 (2002), No. 4, 501-543.

[42] C. S. Patlak. Random walk with persistence and external bias. Bull. Math. Biol. Biophys., 15 (1953), No. 3, 311-338.

[43] B. Perthame, A. -L. Dalibard. Existence of solutions of the hyperbolic Keller-Segel model. Trans. Amer. Math. Soc., 361 (2008), No. 5, 2319-2335.

[44] A. B. Potapov, T. Hillen. Metastability in Chemotaxis Models. J. Dyn. Diff. Eq., 17 (2005), No. 2, 293-330.

[45] R. Schaaf. Stationary solutions of Chemotaxis systems. Trans. Am. Math. Soc., 292 (1985), No. 2, 531-556.

[46] R. Temam, Infinite-Dimensional Dynamical Systems in Mechanics and Physics. SpringerVerlag, New York, 1988.

[47] J. J. L Velázquez. Point dynamics in a singular limit of the Keller-Segel model 1: motion of the concentration regions. SIAM J. Appl. Math., 64 (2004), No. 4, 1198-1223.

[48] M. Winkler. Does a volume filling effect always prevent chemotactic colapse. Math. Meth. Appl. Sci., 33 (2010), No. 1, 12-24.

[49] Z.A. Wang, T. Hillen. Classical solutions and pattern formation for a volume filling chemotaxis model. Chaos., 17 (2007), No. 3, 037108-037121. 
[50] D. Wrzosek. Global attractor for a chemotaxis model with prevention of overcrowding. Nonlinear Anal. TMA., 59 (2004), No. 8, 1293-1310.

[51] D. Wrzosek. Long time behaviour of solutions to a chemotaxis model with volume filling effect. Proc. Roy. Soc. Edinburgh., 136A (2006), No. 2, 431-444.

[52] D. Wrzosek. Chemotaxis models with a threshold cell density. in: Parabolic and NavierStokes equations. Part 2, 553-566, Banach Center Publ., 81, Warsaw, 2008.

[53] D. Wrzosek. Model of chemotaxis with threshold density and singular diffusion. Nonlinear Anal. TMA.. to appear.

[54] Y. Zhang, S. Zheng. Asymptotic Behavior of Solutions to a Quasilinear Nonuniform Parabolic System Modelling Chemotaxis. J. Diff. Equations. in press. 